Neue Cochrane-Analyse

\title{
Halbiert Vitamin D das Risiko schwerer Asthma-Exazerbationen?
}

\author{
Vitamin D scheint sich immer mehr zum Universalheilmittel zu entwickeln. Eine neuen \\ Analyse der Cochrane Library zufolge reduziert die regelmäßige Einnahme das Risiko \\ schwerer Asthma-Attacken.
}

\begin{abstract}
- Niedrige Vitamin-D-Spiegel sind mit einem erhöhten Risiko von Asthma-Anfällen bei Kindern und Erwachsenen assoziiert. Möglicherweise hilft VitaminD-Supplementierung, Atemwegsinfekte zu verhüten, die wiederum ein Asthma bronchiale verschlechtern können. Doch wie ist die Datenlage zur Frage, ob Vitamin-D-Einnahme Asthma-Anfälle verhindert?

Das Cochrane Autorenteam fand bis dato sieben Studien mit zusammen 435 Kindern sowie zwei Studien mit 658 Er-
\end{abstract}

wachsenen mit einer Asthma-Erkrankung überwiegend leichter bis moderater Ausprägung. Untersucht wurde der Effekt einer 6- bis 12-monatigen Vitamin-D-Einnahme zusätzlich zur gewohnten antiasthmatischen Medikation.

Die Autoren fanden heraus, dass Asthmatiker durch Vitamin-D-Einnahme ihr Risiko einer schweren Asthmaattacke, die eine Behandlung im Krankenhaus oder bei einem Notarzt erforderte, von $6 \%$ auf $3 \%$ reduzierten. Unter Vitamin D war auch das Risiko einer oralen Steroideinnahme geringer. Diese Unterschiede wurden v. a. bei erwachsenen Patienten beobachtet. Auf Lungenfunktion oder tägliche Symptomatik hatte das Vitamin D keinen Einfluss.

Auch wenn die ERS von einer "high quality evidence" spricht, scheint angesichts der geringen Patientenzahlen noch etwas Vorsicht angebracht. Schaden tut das Vitamin D indes nicht.

\section{Dr. med. Dirk Einecke}

- Quelle: International Congress 2016 der European Respiratory Society ERS, London, 3.-7. September 2016

\section{Frühe Menopause erhöht Risiko für Lungenerkrankungen}

Frühe Menopause sowie Verlust von Gebärmutter und Ovarien sind assoziiert mit einem akzelerierten Abfall der Lungenfunktion.

Beim europäischen Lungenkongress ERS 2016 berichteten Autoren des Imperial College in London über eine von der British Lung Foundation unterstützte Studie mit 140.000 Frauen, bei denen sowohl Daten über die Lungenfunktion als auch Informationen über den Menopausen-Status vorlagen. Sie untersuchten Assoziationen zwischen den Lungenfunktionsparametern $\mathrm{FEV}_{1}, \mathrm{FVC}$, der spirometrischen Restriktion (FVC $<\mathrm{LLN}$ ) sowie der Atemwegsobstuktion ( $\left.\mathrm{FEV}_{1} / \mathrm{FVC}<\mathrm{LLN}\right)$, setzten dies in Beziehung zum menopausalen Status bzw. dem Alter bei der Menopause und schlossen den Einfluss des Rauchens statistisch aus.

In der "mit Abstand größten Studie zu dieser Fragestellung" stellte sich heraus, dass Frauen schon mit einer natürlichen Menopause einen deutlichen Verlust der Lungenfunktion erfahren. Ihr FVC war im Schnitt um $42 \mathrm{ml}$ niedriger und ihr FEV 1 im Schnitt um $34 \mathrm{ml}$ niedriger als bei prämenopausalen Frauen. Die Wahrscheinlichkeit für eine im Altersdurchschnitt niedriger Lungenfunktion war um 27\% erhöht, nach Hysterektomie um 31-36\%.

Frauen mit einer Menopause vor dem 45. Lebensjahr wiesen im Alter über 60 Jahren ein um 20\% erhöhtes Risiko für eine erniedrigte Lungenfunktion auf als Frauen mit einer Menopause im Alter von 48-53 Jahren.

- Quelle: International Congress 2016 der European Respiratory Society ERS, London, 3.-7. 9. 2016

\section{Häufiges Putzen kann den Lungen schaden}

Die beim Putzen eingeatmeten Dämpfe der Putzmittel haben offensichtlich respiratorische Relevanz. Bisher war bereits bekannt, dass Beschäftigte im Putzdienst zu einer der Berufsgruppen mit dem höchsten COPD-Risiko gehören.

Nun berichten Autoren aus Norwegen über die Langzeitwirkungen der chronischen Putzmittelexposition. Sie hatten im Rahmen des European Community Respiratory Health Survey (ECRHS) über 20 Jahre die Lungenfunktion von knapp 3.500 Frauen überwacht. Nach Adjustierung für Störvariable wiesen Frauen ohne Putzaktivitäten jährliche Abfällen der Lungenfunktionsparameter FVC und $\mathrm{FEV}_{1}$ von 19,5 ml und 29,6 ml auf. Bei Frauen, die regelmäßig zuhause reinemachten, fielen diese Wert jährlich um 24,6 $\mathrm{ml}$ und 33,6 $\mathrm{ml}$ ab. Hauptberufliche Putzfrauen wiesen eine durchschnittliche jährliche FVC- und FEV 1 -Verluste von $27,6 \mathrm{ml}$ und $34,6 \mathrm{ml}$ auf. Die Unterschiede im Vergleich zu Frauen ohne Putzaktivitäten waren signifikant.

Fazit der Autoren: Wer regelmäßig putzt, weist ein erhöhtes Risiko für obstruktive bzw. restriktive Lungenerkrankungen auf. Betroffene sollten den Arzt aufsuchen, wenn sie Atembeschwerden verspüren. Putzsprays sollten mit Vorsicht eingesetzt werden. 